'Escuela de Tecnología Médica, Universidad Austral de Chile, Sede Puerto Montt, Chile. 2Departamento de Salud Pública y Centro de Excelencia CIGES, Universidad de la Frontera, Temuco, Chile. aMagíster en Epidemiología Clínica, Tecnólogo Médico. 'Bioestadístico. 'Doctor en Salud Pública.

Recibido el 25 de noviembre de 2015, aceptado el 11 de junio de 2016.

Correspondencia a:

Marco Barra Mora Los Pinos s/n. Balneario Pelluco, Puerto Montt. mbarra@spm.uach.cl

\section{Desigualdad en la prevalencia de parasitosis intestinal en escolares de una escuela urbana y dos rurales de la comuna de Puerto Montt}

\author{
MARCO BARRA ${ }^{1, \mathrm{a}}$, LUIS BUSTOS ${ }^{2, \mathrm{~b}}$, XIMENA OSSA $^{2, \mathrm{c}}$
}

\begin{abstract}
Background: Parasitic infections in children reflect social inequalities throughout the world, especially in urban and rural territories. Aim: To assess inequalities in the prevalence of infections by protozoa and intestinal helminths and associated factors in children of different geographical areas from the commune of Puerto Montt. Material and Methods: Cross-sectional study carried out in 103 students from one urban and two rural schools in the commune of Puerto Montt. A socio-demographic survey was applied and the presence parasitic infections was evaluated in serial stool samples. Results: The prevalence of Protozoan and intestinal helminthic infections were 37.5 and $68.1 \%$ in urban and rural areas respectively. A regression analysis showed that the differences between rural and urban children, disappear when the results are adjusted by family income and the quality of sewage disposal. In urban children, the prevalence of parasitic infections increases along with the decrease in family income. Conclusions: Income and sanitary conditions rather than being or urban or rural origin explain the variations in rates of childhood parasitic infections. Infections with protozoa and intestinal helminths are an indicator of social inequality in health.
\end{abstract}

(Rev Med Chile 2016; 144: 886-893)

Key words: Intestinal Diseases; Parasitic Diseases; Prevalence; Socioeconomic Factors.

\section{I}

as desigualdades sociales en salud se refieren a los efectos que tienen factores como la clase social, educación, vivienda, etnia y distribución geográfica, entre otros, sobre el estado de salud de una población ${ }^{1}$. América Latina y el Caribe son las regiones del mundo que presentan una importante desigualdad social, además de graves dificultades en aspectos de salud y de acceso a servicios atingentes, contribuyendo al aumento de la disparidad en salud entre grupos sociales y regiones geográficas ${ }^{2}$. Chile no está libre de este problema, clasificándose entre los países con mayor desigualdad en el mundo ${ }^{3}$. Las infecciones parasitarias son enfermedades asociadas a condiciones de desigualdad social, siendo más prevalentes en contextos de pobreza, hacinamiento, deficientes condiciones sanitarias, bajo nivel educacional y residencia en cordones urbano marginales y rurales.

Múltiples estudios identifican factores sociales, económicos, culturales y sanitarios asociados a parasitosis intestinal en niños ${ }^{5,6}$. Una parte importante de estos estudios se realizan en asentamientos rurales o semirurales, donde estas condiciones son más bien deplorables. Estas patologías representan 
un problema de salud pública a nivel mundial, afectando especialmente la salud de la población infantil, causando potenciales deficiencias en el aprendizaje, problemas cognitivos y retardo en el desarrollo pondo-estatural ${ }^{7,8}$, que finalmente repercuten en todo el ciclo vital.

En Chile, los estudios de parasitosis intestinal en niños en la última década son escasos. Mediciones realizadas en el norte del país, en asentamientos rurales y urbanos, muestran una prevalencia que oscila entre $70 \%$ a $80 \%$, cuyas especies parasitarias se relacionan con el lugar geográfico y climático,10. En la zona central, la prevalencia encontrada fue de $55 \%$ en un poblado semirural de la Región Metropolitana y de 76\% en una zona rural de la región del Maule ${ }^{11,12}$. En el sur del país, estudios de la década de los 90, en zonas rurales, muestran prevalencias semejantes a las anteriores ${ }^{13-15}$, asociándose a deficientes condiciones higiénicas, escasa infraestructura sanitaria y pobreza a esta realidad.

Existe evidencia que la prevalencia de parasitosis disminuye según mejoran las condiciones sanitarias y socio-económicas de los territorios ${ }^{16,17}$. Chile, en los últimos años ha mejorado sus indicadores sociales y económicos, sin embargo, en aspectos de desigualdad social el avance ha sido más lento ${ }^{18}$, por lo que las bondades del progreso no han llegado a todos los territorios por igual. Puerto Montt, capital de la Región de Los Lagos, ha triplicado y duplicado sus gastos en educación y salud, respectivamente, en la última década y ha sido una de las comunas con mayor crecimiento y desarrollo en dicho período ${ }^{19,20}$, no obstante, es la zona urbana la que se ha visto favorecida preferentemente de los beneficios del desarrollo. Poco se conoce de aquellas enfermedades que potencialmente podrían marcar desigualdades sociales en salud en sectores que quedan rezagados del surgimiento económico.

El objetivo de este estudio es evaluar desigualdades en la prevalencia de infección por protozoos $y$ helmintos intestinales en niños y niñas de zonas geográficas distintas de un territorio común (comuna de Puerto Montt) y los factores asociados a esta condición.

\section{Material y Método}

Estudio de corte transversal realizado en tres escuelas de la comuna de Puerto Montt: Escuela Chiloé (matrícula de 491 estudiantes) ubicada en un sector populoso de la ciudad, y dos escuelas rurales ubicadas en los sectores de Lenca (165 estudiantes) y Chamiza (215 estudiantes). El caserío de Chamiza y la aldea de Lenca se ubican en el kilómetro 9 y 33 de la Carretera Austral de Chile respectivamente, con poblaciones pequeñas que no superan las 720 personas entre ambas ${ }^{21}$. En ambos sectores, la principal actividad económica es la pesca artesanal y el turismo rural.

Se seleccionó a la totalidad de estudiantes que cursaban primero y segundo básico en las tres escuelas durante el año 2013 (122 alumnos). El estudio y procedimientos fueron explicados a los niños en presencia de las profesoras encargadas de los respectivos cursos, se solicitó su asentimiento en primera instancia, y después, el consentimiento informado a padres y apoderados, junto con las instrucciones de la toma de muestra.

Se aplicó una encuesta conteniendo datos personales y del ámbito socio-económico y demográfico con preguntas extraídas del módulo familiar y de vivienda de la Encuesta Sobre Calidad de Vida y Salud 2000 y 2006 y el resultado parasitológico. El procedimiento se realizó a través de exámenes parasitológicos seriados de deposiciones (días no consecutivos), utilizando como fijador fenol, alcohol, formalina y suero fisiológico (PAFS). El procesamiento se efectuó a través del método de Burrows ${ }^{22}$ y la observación en microscopio óptico con aumento de 10, 40 y 100X, por un solo observador. Una copia del informe de los resultados fue entregada en sobre sellado a cada apoderado, con una pequeña explicación y recomendación de asistir a su centro de atención de salud, cuando éstos fueron positivos.

Se realizó análisis descriptivo de los datos estimando diferencias según procedencia urbano o rural a través de test, test exacto de Fisher y Prueba de Tendencia de Cuzick $(\mathrm{p}<0,05)$ según tipo de variable. Se estimó magnitud, dirección de asociación, interacción y confusión a través de regresión logística. La desigualdad en la prevalencia de parasitosis se estimó a través del Índice de $\mathrm{Efecto}^{23}$. Se utilizó el paquete estadístico STATA, versión 12.0.

Este estudio contó con la aprobación del Comité de Ética Científica de la Universidad de La Frontera.

\section{Resultados}

De los 122 estudiantes, 19 se negaron a participar $(15,5 \%)$, por lo que el grupo de estudio 
quedó conformado por 103 estudiantes. La edad promedio de los estudiantes fue de 6,7 años, con un mínimo de 6 y un máximo de 9 años, encontrándose una edad levemente mayor entre los niños urbanos $(\mathrm{p}=0,022)$. El promedio de años de escolaridad materna fue de 10 años, con un mínimo de 1 y máximo 17. El promedio de integrantes del hogar fue de 5 personas con un mínimo de 2 y un máximo de 10; la mayoría perteneciente al Sistema Público de Salud, siendo más frecuente el nivel FONASA A $(67,2 \%)$ y B $(19,4 \%)$. En estas características no se observó diferencias significativas según lugar de procedencia. Las características sociodemográficas del grupo que rehusó participar no tuvieron diferencias significativas con el grupo de estudio.

En general, se observó mayor proporción de hombres, con una distribución levemente mayor en el sector urbano, y una mayor frecuencia de familias que perciben menos de $\$ 199.999$ mensuales como ingreso familiar. Se observa que la principal fuente de agua corresponde a la red pública, la mayor proporción se encuentra conectada a alcantarillado y elimina la basura a través del camión recolector (Tabla 1).

$\mathrm{Al}$ analizar características sociodemográficas y sanitarias según lugar de procedencia, se observó que no existen diferencias significativas en sexo, sistema de salud e ingreso familiar. Se encontraron diferencias significativas en las fuentes de agua, disposición de excretas y disposición de basura $(\mathrm{p}<0,05)$.

En la Tabla 1 se observa que la distribución de parasitosis intestinal es significativamente mayor en los niños del sector rural, evidenciando un riesgo tres veces mayor en el análisis bivariado. No existen diferencias significativas de parasitismo por sexo ni por diferencias en el ingreso familiar, sin embargo, al realizar análisis de tendencia del riesgo de infección parasitaria según ingreso familiar, se evidencia un aumento significativo del riesgo conforme disminuye dicho ingreso $(\mathrm{p}=0,024)$.

Tabla 1. Caracterización, distribución y riesgo de la infección parasitaria, en la comuna de Puerto Montt

\begin{tabular}{|c|c|c|c|c|c|}
\hline & $\begin{array}{l}\text { Total } \\
\text { n (\%) }\end{array}$ & $\begin{array}{c}\text { No parasitado } \\
\text { n (\%) }\end{array}$ & $\begin{array}{c}\text { Parasitado } \\
\text { n (\%) }\end{array}$ & OR & IC (95\%) \\
\hline \multicolumn{6}{|l|}{ Procedencia* } \\
\hline Urbano & $56(54,4)$ & $35(62,5)$ & $21(37,5)$ & - & \\
\hline Rural & $47(45,6)$ & $15(31,9)$ & $32(68,1)$ & 3,6 & $1,6-8,1$ \\
\hline \multicolumn{6}{|l|}{ Sexo } \\
\hline Mujer & $50(48,5)$ & $24(48)$ & $26(52)$ & - & \\
\hline Hombre & $53(51,5)$ & $26(49,1)$ & $27(50,9)$ & 0,9 & $0,4-2,1$ \\
\hline \multicolumn{6}{|l|}{ Ingreso familiar** } \\
\hline Más de $\$ 400.000$ & $6(5,8)$ & $4(66,7)$ & $2(33,3)$ & - & \\
\hline$\$ 300.000-\$ 399.999$ & $14(13,6)$ & $9(64,3)$ & $5(35,7)$ & 1,1 & $0,1-8,4$ \\
\hline$\$ 200.000-\$ 299.999$ & $36(34,9)$ & $20(55,6)$ & $16(44,4)$ & 1,6 & $0,3-9,9$ \\
\hline Menos de \$199.999 & $47(45,7)$ & $17(36,2)$ & $30(63,8)$ & 3,5 & $0,6-21,3$ \\
\hline \multicolumn{6}{|l|}{ Fuente de agua } \\
\hline Red Pública & $89(86,4)$ & $44(49,4)$ & $45(50,6)$ & - & \\
\hline Sin red Pública & $14(13,6)$ & $6(42,7)$ & $8(57,1)$ & 1,3 & $0,4-4,1$ \\
\hline \multicolumn{6}{|l|}{ Disp. de excretas*** } \\
\hline Conectado a alcantarillado & $57(55,3)$ & $34(59,7)$ & $23(40,3)$ & - & \\
\hline Conectado a fosa séptica & $36(35,0)$ & $13(36,1)$ & $23(63,9)$ & 2,6 & $1,1-6,2$ \\
\hline Otro & $10(9,7)$ & $3(30,0)$ & $7(70,0)$ & 3,4 & $0,8-14,7$ \\
\hline \multicolumn{6}{|l|}{ Disposición de basura } \\
\hline Camión recolector & $99(96,1)$ & $48(48,5)$ & $51(51,5)$ & - & \\
\hline Quema & $4(3,9)$ & $2(50)$ & $2(50)$ & 0,9 & $0,1-6,9$ \\
\hline Total & $103(100)$ & $50(48,5)$ & $53(51,5)$ & & \\
\hline
\end{tabular}

${ }^{*} p=0,002 .{ }^{* *}$ Prueba de Tendencia de Cuzick, $p=0,024 .{ }^{* * *}$ Prueba de Tendencia de Cuzick, $p=0,016$. 
En la Tabla 1 también se evidencia que, respecto de las condiciones sanitarias, no existen diferencias significativas de prevalencia de infección según fuente de agua potable y disposición de basura. Se muestra una mayor proporción de niños parasitados en los grupos que tienen mayor deficiencia en el sistema de eliminación de excretas $(p=0,04) y$, a su vez, una tendencia significativa a aumentar el riesgo de infección en la medida que el sistema de disposición de excretas es más precario.

En la Tabla 2 se muestra la distribución de protozoos y helmintos intestinales, destacándose como protozoos más frecuentes Endolimax nana y Blastocystis hominis en ambos sectores. En menor

Tabla 2. Distribución de protozoos y helmintos intestinales según procedencia de los estudiantes, en la comuna de Puerto Montt

\begin{tabular}{|lcc|}
\hline Taxon parasitario & \multicolumn{2}{c|}{ Procedencia } \\
& $\begin{array}{c}\text { Urbano } \\
\text { n (\%) }\end{array}$ & $\begin{array}{c}\text { Rural } \\
\text { n (\%) }\end{array}$ \\
Protozoos & & \\
$\quad$ Endolimax nana & $9(42,9)$ & $12(37,5)$ \\
Blastocystis hominis & $7(33,3)$ & $13(40,6)$ \\
Entamoeba coli & $4(19,0)$ & $5(15,7)$ \\
Chilomastix mesnili & $1(4,8)$ & 0 \\
$\quad$ Giardia lamblia & 0 & $1(3,1)$ \\
Helmintos & & 1 \\
$\quad$ Ascaris lumbricoides & 0 & $32(100)$ \\
Total & $21(100)$ & $3,1)$ \\
\hline
\end{tabular}

proporción y sólo en el sector rural se encontraron Giardia lamblia y Ascaris lumbricoides, como patógenos. Del total de niños, 28,3\% resultaron infectados por más de un parásito y/o comensal.

En el análisis de interacción entre procedencia y las variables ingreso familiar y disposición de excretas, a través de regresión logística, no existe modificación de efecto, sin embargo, al ajustar por ambas variables se produce sesgo de confusión, por lo que es necesario ajustar por ambas variables la asociación entre parasitosis y lugar de residencia. Esto muestra que el riesgo de mayor infección en el sector rural (OR = 3,6 IC 95\% 1,6-8,1) se pierde cuando se ajusta por ingreso familiar y disposición de excretas (Tabla 3 ).

Con respecto al análisis de desigualdad en la prevalencia de infección parasitaria entre escolares urbanos y rurales según ingreso familiar, a través de la determinación del índice de efecto, se observó que en los niños del sector urbano, en promedio, al disminuir el ingreso familiar mensual en 100 unidades (M\$), aumenta 53,2\% la prevalencia de infección parasitaria, a diferencia del sector rural, que en las mismas condiciones de disminución del ingreso, aumenta en menor proporción $(43,8 \%)$.

\section{Discusión}

La prevalencia de parasitosis intestinal fue mayor en escolares del sector rural de Puerto Montt $(68,1 \%)$ que en los urbanos $(37,5 \%)$, por

Tabla 3. Asociación entre presencia de protozoos y helmintos intestinales y procedencia, ajustado por ingreso familiar y disposición de excretas, en la comuna de Puerto Montt

\begin{tabular}{|c|c|c|c|c|c|}
\hline & n casos & OR & IC ( & 5\%) & $\mathbf{p}$ \\
\hline $\begin{array}{l}\text { Urbano } \\
\text { Rural }\end{array}$ & $\begin{array}{l}21 \\
32\end{array}$ & - & 0,9 & 84,3 & 0,065 \\
\hline $\begin{array}{l}\text { Ingreso familiar } \\
\text { Más de } \$ 400.000 \\
\$ 300.000-\$ 399.999 \\
\$ 200.000-\$ 299.999 \\
\text { Menos de } \$ 199.999\end{array}$ & $\begin{array}{r}2 \\
5 \\
16 \\
30\end{array}$ & $\begin{array}{l}- \\
1,3 \\
1,8 \\
3,8\end{array}$ & $\begin{array}{l}0,2 \\
0,3 \\
0,6\end{array}$ & $\begin{array}{l}10,7 \\
12,3 \\
25,3\end{array}$ & $\begin{array}{l}0,794 \\
0,526 \\
0,167\end{array}$ \\
\hline $\begin{array}{l}\text { Disposición de excretas } \\
\text { Conectado a alcantarillado } \\
\text { Conectado a fosa séptica } \\
\text { Otro }\end{array}$ & $\begin{array}{r}23 \\
23 \\
7\end{array}$ & $\begin{array}{c}- \\
0,4 \\
0,3\end{array}$ & $\begin{array}{l}0,03 \\
0,02\end{array}$ & $\begin{array}{l}3,6 \\
4,5\end{array}$ & $\begin{array}{l}0,380 \\
0,409\end{array}$ \\
\hline
\end{tabular}


lo tanto, se evidencia desigualdad geográfica. Los ingresos familiares y el sistema de disposición de excretas fueron factores que incidieron en un mayor riesgo de infección en general. Ambos factores son los que finalmente modulan el riesgo de infección intestinal por protozoos y helmintos en estos estudiantes; el lugar de residencia deja de ser significativo al ajustar por estas variables, por lo tanto, también se demuestran desigualdad social o inequidad en salud en la prevalencia de estas patologías.

La fuente de agua y la recolección de basuras no tienen mayor relevancia ya que a pesar de haber diferencias en estos factores en lo rural y urbano, los sistemas alternativos existentes en los territorios rurales son bastante efectivos. Los principales protozoos en ambos lugares fueron Endolimax nana y Blastocystis hominis. Si bien, ambos se consideran comensales, su asociación a cuadros de diarrea crónica o enterocolitis, han hecho discutible este rol sobre todo en presencia de otros parásitos o en cuadros de inmunodepresión $^{24-26}$. Por otro lado, el mecanismo de transmisión de estos protozoos, así como también de otros comensales es el fecalismo ${ }^{24}$, lo que indica que las condiciones higiénico-sanitarias son deficientes en estas familias.

Un hallazgo interesante en el análisis de desigualdad es que los estudiantes urbanos tienen una probabilidad de prevalencia mayor de parasitosis intestinal que los estudiantes rurales cuando los ingresos familiares disminuyen.

Las prevalencias encontradas en este estudio en el sector rural se acercan a otros estudios realizados en Chile en territorios similares ${ }^{9,11,12}$ y en América Latina y el Caribe (ALC) ${ }^{27-29}$, diferenciándose de otros que declaran prevalencias sobre $90 \%{ }^{30}$, donde incide la condición étnica además de pobreza e insalubridad. En el sector urbano, la prevalencia encontrada en este estudio es más baja que en el sector rural y menor que la reportada por Araya et al. (2009) en Antofagasta ${ }^{10}$, no encontrándose otros estudios en sectores urbanos con los que comparar, por lo menos en esta última década. En ALC abundan estudios en sectores semiurbanos $y$ urbanos en donde las prevalencias encontradas son variadas ${ }^{5,31,32}$, reportándose valores desde $28 \%$ hasta $83 \%$ aproximadamente, siendo la pobreza y la falta de estructura sanitaria los factores comunes asociados a esta condición. Similares reportes son encontrados en países africanos y asiáticos, donde las prevalencias en niños generalmente son altas, entre $66 \%$ a $98 \%$ aproximadamente en la mayoría de los estudios ${ }^{33,34}$, pero también se reportan prevalencias menores ${ }^{35}$.

En general, cuando se compara la frecuencia de parasitosis intestinal en niños en áreas rurales y urbanas de un territorio los resultados son diversos. En algunos lugares las prevalencias son similares, diferenciándose más bien en las especies descubiertas ${ }^{36}$; en otros territorios, la prevalencia encontrada en las zonas rurales es notablemente más alta que en las zonas urbanas ${ }^{37,38}$, mientras que en otros, los sectores urbanos son los más afectados $^{39,40}$. Todos los estudios relacionan determinantes sociales con mayor o menor prevalencia de parásitos y/o comensales intestinales y Chile no puede retrotraerse a esta realidad. Aunque existe evidencia que en los últimos 20 años, la pobreza ha disminuido gradualmente tanto en zonas urbanas como rurales, en este último sector, la variabilidad encontrada entre los territorios es mucho mayor. Es así, que en la zona sur de Chile, se pueden encontrar territorios rurales con $2 \%$ de su población en situación de pobreza y otros con más de $30 \%$ en esta condición, acompañado de bajo nivel educacional y condiciones sanitarias más precarias ${ }^{41}$.

En este estudio no se encontró diferencias significativas en la prevalencia de protozoos y/o helmintos intestinales, según años de educación de la madre, hacinamiento, fuente de agua y disposición de basura, a diferencia de muchos otros estudios $^{28,31,42,43}$. Esto puede explicarse porque, en promedio, los años declarados de educación materna fueron mayores que en la mayoría de los estudios que sólo alcanzan a primer ciclo básico; las fuentes de agua provienen de la red de agua potable o de una cooperativa de agua potable rural, por lo tanto de buena calidad; por otro lado, la Municipalidad de Puerto Montt concesionó el servicio de extracción de basura a través de contenedores en todo el radio urbano y rural de la comuna ${ }^{44}$. Sin embargo, se encontró asociación entre parasitosis infantil y disposición de excretas y, además, una tendencia significativa a mayor riesgo de infección en estudiantes que tienen sistemas de eliminación de excretas más precarios, esto concuerda con lo descrito por la mayor parte de los estudios publicados sobre el tema, por lo que podría suponerse que el problema principalmente radicaría en falla de medidas hi- 
giénicas que previenen la contaminación oro-fecal y la contaminación de alimentos.

El aporte más importante de este estudio es demostrar que el lugar de procedencia, por sí solo, no constituye una variable significativa que permita dar cuenta de un mayor riesgo de parasitosis, sino que las condiciones sanitarias y de vida que puede aportar el mayor ingreso familiar son las que lo determinan. Tanto es así, que la determinación del índice de efecto, demostró que, a iguales condiciones de disminución del ingreso familiar, los niños del sector urbano tienen una mayor probabilidad de infección parasitaria con respecto a los niños rurales. Este resultado podría potencialmente explicarse porque los niños de las zonas rurales estarían más expuestos y esta condición permitiría desarrollar un efecto inmunológico protector contra la infección parasitaria, hipótesis que aún está en estudio ${ }^{45}$.

Actualmente, la disminución de las brechas en salud es una meta perseguida en todo el mundo. Una primera etapa consiste en la identificación y medición de las desigualdades, así como también de sus determinantes ${ }^{46}$. Este estudio demuestra que la prevalencia de parasitosis intestinal en escolares podría utilizarse como un indicador de inequidad en salud.

Por último, resulta necesario señalar algunas limitaciones que deben considerarse para evaluar estos resultados tales como el pequeño tamaño de la población de estudio y la falta de medición de otras variables como hábitos higiénicos, tenencia de mascotas y características de la vivienda, entre otras, que podrían haber aportado un poco más de antecedentes al análisis de los datos, aunque, probablemente no hubieran cambiado lo fundamental.

Se sugiere realizar nuevos estudios que permitan seguir profundizando respecto de la distribución de la parasitosis intestinal en los territorios del país, ojalá incorporando intervenciones, porque las prevalencias detectadas no son menores y aún existen desigualdades sociales dentro y entre territorios que determinan diferencias en la salud de las personas, especialmente los niños.

\section{Referencias}

1. Organización Mundial de la Salud. Subsanar las desigualdades en una generación: Alcanzar la equidad sanitaria actuando sobre los determinantes sociales de la salud. 2008. Available from: http://apps.who.int/iris/ handle/10665/44084 [consultado el 28 de octubre de 2015].

2. Casas JA, Dachs JN, Bambas A. Health disparities in Latin America and the caribbean: the role of social and economic determinants. In: Equitiy and Health Views from the Pan American Sanitary Bureau. PAHO; 2001. p. 22-49. Available from: http://citeseerx.ist.psu.edu/ viewdoc/download?doi=10.1.1.409.5912\&rep=rep1\&type $=$ pdf\#page $=31$ [consultado el 28 de octubre de 2015].

3. Pnud. Informe sobre Desarrollo Humano 2014. Available from: http://hdr.undp.org/sites/default/files/hdr14report-en-1.pdf [consultado el 28 de octubre de 2015].

4. Gazzinelli A, Correa-Oliveira R, Yang G-J, Boatin BA, Kloos H. A research agenda for helminth diseases of humans: social ecology, environmental determinants, and health systems. PLoS Negl Trop Dis 2012; 6 (4): e1603.

5. Garbossa G, Pía Buyayisqui M, Geffner L, López Arias L, de la Fournière S, Haedo AS, et al. Social and environmental health determinants and their relationship with parasitic diseases in asymptomatic children from a shantytown in Buenos Aires, Argentina. Pathog Glob Health 2013; 107 (3): 141-52.

6. Berrilli F, Di Cave D, N’Guessan R, Kaboré Y, Giangaspero A, Sorge RP, et al. Social determinants associated with Giardia duodenalis infection in southern Côte d'Ivoire. Eur J Clin Microbiol Infect Dis 2014; 33 (10): 1799-802.

7. Nguyen NL, Gelaye B, Aboset N, Kumie A, Williams MA, Berhane $Y$. Intestinal parasitic infection and nutritional status among school children in Angolela, Ethiopia. J Prev Med Hyg 2012; 53 (3): 157-64.

8. Simsek Z. Effect of Giardia Infection on Growth and Psychomotor Development of Children Aged 0-5 Years. J Trop Pediatr 2004; 50 (2): 90-3.

9. Bórquez C, Lobato I, Montalvo MT, Marchant P, Martínez P. Enteroparasitosis en niños escolares del valle de Lluta, Arica-Chile. Parasitol latinoam 2004; 59: 175-8.

10. Araya J, Araneda L, Landskron G. Enteroparasitosis en alumnos de educación básica de la ciudad de Antofagasta (2006-2007). Rev Cienc Salud 2009; 13 (1): 33-44.

11. Mercado R, Castillo D, Muñoz V, Sandoval L, Jercic MI, Schenone $\mathrm{H}$, et al. Intestinal protozoa and infections in pre-school and elementary school-children from colina county, Santiago, Chile, 2003. Parasitol latinoam 2003; 58 (3-4): 173-6.

12. Vidal FS, Toloza ML, Cancino FB. Evolución de la prevalencia de enteroparasitosis en la ciudad de Talca, 
Región del Maule, Chile. Rev Chil Infectología 2010; 27 (4): 336-40.

13. Mercado R, Otto JP, Musleh M, Pérez M. Human infection by intestinal protozoa and helminths in Calbuco County, X Region, Chile, 1997. Boletín Chil Parasitol 1997; 52 (1-2): 36-8.

14. Navarrete N, Torres P. Prevalence of infection by intestinal helminths and protozoa in school children from a coastal locality in the province of Valdivia, Chile. Boletín Chil Parasitol 1994; 49 (3-4): 79-80.

15. Mejías G. Intestinal parasite infections in rural students of Chiloé archipelago, X Región, Chile. Boletín Chil Parasitol 1993; 48 (1-2): 28-9.

16. Sinniah B, Hassan AKR, Sabaridah I, Soe MM, Ibrahim Z, Ali O. Prevalence of intestinal parasitic infections among communities living in different habitats and its comparison with one hundred and one studies conducted over the past 42 years (1970 to 2013) in Malaysia. Trop Biomed 2014; 31 (2): 190-206.

17. Karagiannis-Voules DA, Biedermann P, Ekpo UF, Garba A, Langer E, Mathieu E, et al. Spatial and temporal distribution of soil-transmitted helminth infection in sub-Saharan Africa: a systematic review and geostatistical meta-analysis. Lancet Infect Dis 2015; 15 (1): 74-84.

18. Ministerio de Desarrollo Social. Informe política social 2013. Available from: http://www.ministeriodesarrollosocial.gob.cl/ipos-2013/ [consultado el 30 de octubre de 2015].

19. Biblioteca del Congreso Nacional de Chile. Reportes estadisticos y comunales, 2013. Available from: http://reportescomunales.bcn.cl/2013/index.php/Puerto_Montt [consultado el 30 de octubre de 2015].

20. Cohen B, Obediente E. Estudio "Ranking de Ciudades Inteligentes en Chile”. 2014. Available from: http://paisdigital.org/wp-content/uploads/2014/06/Ranking-Ciudades-Inteligentes-en-Chile.pdf [consultado el 30 de octubre de 2015].

21. Instituto Nacional de Estadisticas. Chile: Ciudades, Pueblos, Aladeas y Caseríos. 2005. Available from: http:// www.ine.cl/canales/usuarios/cedoc_online/censos/pdf/ censo_2002_publicado_junio_2005.pdf [consultado el 30 de octubre de 2015].

22. Muñoz V, Dorn L, Reyes H. Examen coproparasitológico: evaluación de algunas modificaciones al método de Burrows (PAF). Parasitol al día. 1984; 8: 107-11.

23. Schneider MC, Castillo-Salgado C, Bacallao J, Loyola E, Mujica OJ, Vidaurre M, et al. Medición de las desigualdades de salud. Rev Panam Salud Pública 2002; 12 (6): 371-2.

24. Becerril M. Amibas Comensales. En: Ruíz A, Editora. Parasitología Médica. Editorial McGraw-Hill/Interame- ricana Editores S.A. de C.V.4 Ed.; 2014. p. 51-62.

25. Khalil S, Mirdha B, Sinha S, Panda A, Singh Y, Joseph A. Intestinal Parasitosis in Relation to Anti-Retroviral Therapy, CD4 + T-cell Count and Diarrhea in HIV Patients 2015; 53 (6): 705-12.

26. Graczyk TK, Shiff CK, Tamang L, Munsaka F, Beitin AM, Moss WJ. The association of Blastocystis hominis and Endolimax nana with diarrheal stools in Zambian school-age children. Parasitol Res 2005; 98 (1): 38-43.

27. Cepon-Robins TJ, Liebert MA, Gildner TE, Urlacher SS, Colehour AM, Snodgrass JJ, et al. Soil-transmitted helminth prevalence and infection intensity among geographically and economically distinct Shuar communities iprn the Ecuadorian Amazon. J Parasitol 2014; preprint (5): 598-607.

28. Quihui L, Valencia ME, Crompton DWT, Phillips S, Hagan P, Morales G, et al. Role of the employment status and education of mothers in the prevalence of intestinal parasitic infections in Mexican rural school children. BMC Public Health 2006; 6 (1): 225.

29. Macchioni F, Segundo H, Gabrielli S, Totino V, Gonzales PR, Salazar E, et al. Dramatic Decrease in Prevalence of Soil-Transmitted Helminths and New Insights Into Intestinal Protozoa in Children Living in the Chaco Region, Bolivia. Am J Trop Med Hyg 2015; 92 (4): 794-6.

30. Brandelli CLC, Carli GA De, Macedo AJ, Tasca T. Intestinal parasitism and socio-environmental factors among Mbyá-Guarani indians, Porto Alegre, Rio Grande do Sul, Brazil. Rev Inst Med Trop Sao Paulo 2012; 54 (3): 119-22.

31. Cañete R, Díaz MM, Ávalos García R, Laúd Martínez PM, Manuel Ponce F. Intestinal Parasites in Children from a Day Care Centre in Matanzas City, Cuba. PLoS One 2012; 7 (12): 1-4.

32. Nobre LN, Silva R V, Macedo MS, Teixeira RA, Lamounier JA, Franceschini SCC. Risk factors for intestinal parasitic infections in preschoolers in a low socio-economic area, Diamantina, Brazil. Pathog Glob Health 2013; 107 (2): 103-6.

33. Al-Delaimy AK, Al-Mekhlafi HM, Nasr NA, Sady H, Atroosh WM, Nashiry M, et al. Epidemiology of intestinal polyparasitism among Orang Asli school children in rural Malaysia. PLoS Negl Trop Dis. United States 2014; 8 (8): e3074.

34. Mathewos B, Alemu A, Woldeyohannes D, Alemu A, Addis Z, Tiruneh M, et al. Current status of soil transmitted helminths and Schistosoma mansoni infection among children in two primary schools in North Gondar, Northwest Ethiopia: a cross sectional study. BMC Res Notes 2014; 7 (1): 88.

35. Tulu B, Taye S, Amsalu E. Prevalence and its associated 
risk factors of intestinal parasitic infections among Yadot primary school children of South Eastern Ethiopia: a cross-sectional study. BMC Res Notes 2014; 7 (1): 848.

36. Agbolade OM, Agu NC, Adesanya OO, Odejayi AO, Adigun AA, Adesanlu EB, et al. Intestinal helminthiases and schistosomiasis among school children in an urban center and some rural communities in southwest Nigeria. Korean J Parasitol 2007; 45 (3): 233-8.

37. Fernández MC, Verghese $S$, Bhuvaneswari R, Elizabeth SJ, Mathew T, Anitha A, et al. A comparative study of the intestinal parasites prevalent among children living in rural and urban settings in and around Chennai. J Commun Dis 2002; 34 (1): 35-9.

38. Gamboa MI, Giambelluca LA, Navone GT. Spatial distribution of intestinal parasites in the City of La Plata, Argentina. Medicina 2014; 74 (5): 363-70.

39. Phiri K, Whitty CJ, Graham SM, Ssembatya-Lule G. Urban/rural differences in prevalence and risk factors for intestinal helminth infection in southern Malawi. Ann Trop Med Parasitol 2000; 94 (4): 381-7.

40. Wördemann M, Polman K, Menocal Heredia LT, Junco Díaz R, Collado Madurga AM, Núñez Fernández FA, et al. Prevalence and risk factors of intestinal parasites in Cuban children. Trop Med Int Heal 2006; 11 (12): 181320.

41. Agostini CA, Brown PH, Góngora DP. Distribución
Espacial de la Pobreza en Chile. Estud Econ 2008; 35 (1): 79-110.

42. Soriano SV, Manacorda AM, Pierangeli NB, Navarro MC, Giayetto AL, Barbieri LM, et al. Parasitosis intestinales y su relacion con factores socioeconómicos y condiciones de hábitat en niños de Neuquén, Patagonia, Argentina. Parasitol Latinoam 2005; 60 (3-4): 154-61.

43. Fuentes M, Galíndez L, García D, González N, Goyanes J, Herrera E, et al. Frecuencia de parasitosis intestinales y características epidemiológicas de la población infantil de 1 a 12 años que consultan al Ambulatorio Urbano Tipo II de Cerro Gordo. Barquisimeto, estado Lara. Enero-junio 2007. Kasmera 2011; 39 (1): 31-42.

44. Municipalidad de Puerto Montt. Extracción Residuos Sólidos Domiciliarios Rural. Available from: http:// www.puertomontt.cl/servicios/otros-servicios/retiro-de-basura/extraccion-residuos-solidos-domiciliarios-rural/ [consultado el 3 de noviembre de 2015].

45. Anthony RM, Rutitzky LI, Urban JF, Stadecker MJ, Gause WC. Protective immune mechanisms in helminth infection. Nat Rev Immunol 2007; 7 (12): 975-87.

46. Alleyne GAO, Castillo-Salgado C, Schneider MC, Loyola E, Vidaurre M. Overview of social inequalities in health in the Region of the Americas, using various methodological approaches. Rev Panam Salud Pública 2002; 12 (6): 388-97. 